\title{
Editorial
}

\section{The changing pattern of antibiotic resistance of Neisseria gonorrhoeae}

The World Health Organisation has just issued the conclusions of a consultation on sexually transmitted diseases treatment strategies. ${ }^{1}$ This replaces the previous consultative report issued in $1983 .{ }^{2}$ The recommendations for the treatment of gonorrhoea in these two reports are very different. In 1983 the recommended regimen was either single dose therapy with an injectable or oral penicillin combined with probenecid, or a course of a tetracycline. Spectinomycin or cefoxitin were recommended as alternatives where penicillinase producing Neisseria gonorrhoeae (PPNG) or other resistant gonococci were known to be prevalent. Resistance was treated very much as an emergency issue with some recognised problem areas in South East Asia and Africa.

Now, seven years later gonococcal resistance to antibiotics is a problem in many parts of the world. The new recommended standard regimens are single dose ceftriaxone $250 \mathrm{mg}$ i.m., ciprofloxacin $500 \mathrm{mg}$ oral, or spectinomycin $2 \mathrm{~g}$ i.m. Tetracyclines and oral or injectable penicillins are now only recommended where the level of resistance to these agents is known to be low.

Despite their relatively high initial cost, these three regimens were proposed on the principle that the overall cost of cheaper, less effective regimens (including relapse, complications, further spread and increasing resistance) would in fact be greater. The ceftriaxone and ciproflaxacin doses are higher than those used in some quarters, but it was felt that the higher doses would be more effective in preventing the emergency and spread of resistance to both agents.

Antimicrobial resistance in $N$ gonorrhoeae may be controlled by genes on either plasmid or chromosome. Clinically significant chromosomal resistance to penicillins and tetracyclines results from the additive effect of mutations at several resistance loci, for example, pen $A$, pen $B$, tet, $m t r .{ }^{34}$ With penicillins chromosomal resistance is expressed through changes to the gonococcal cell envelope. ${ }^{5}$ Unfortunately there is no simple rapid test for detecting such resistance. Resistance to other useful antigonococcal compounds is also controlled by chromosomal genes. These include cephalosporins, spectinomycin, thiamphenicol and earlier quino- lones such as rosoxacin. ${ }^{3}$ Resistance to the newer quinolones such as enoxacin and ciprofloxacin is now being seen. ${ }^{6-8}$ This too is presumed to be under chromosomal control. In general chromosomal resistance spreads more slowly than plasmid-mediated resistance. Nevertheless penicillin and tetracycline resistance is now common in many parts of the world with spectinomycin resistance posing a problem in some parts of the Pacific.

Plasmid-mediated resistance to penicillins was first reported in $1976 .{ }^{9}{ }^{10}$ Two plasmids of 3.2 and 4.4 megadaltons were involved originally, those appearing to originate in gonococcal strains from West Africa and the Far East respectively. Although these remain the most common, other penicillinase controlling plasmids have since been described. None is self-transmissable, requiring the presence of a 24.5 megadalton conjugative plasmid to enable them to move between strains. All control the production of the same TEM-1 beta lactamase. Fortunately gonococcal beta lactamase can be detected simply and rapidly. This makes screening for penicillinase producing $N$ gonorrhoeae (PPNG) easy and facilitates epidemiological monitoring of these strains.

In 1985 a second form of plasmid-mediated antibiotic resistance was found to give rise to high level tetracycline resistant $N$ gonorrhoeae (TRNG). The tetracycline resistance plasmid is large, 25.2 megadaltons, and self-transmissable. ${ }^{11}$ Although TRNG were first encountered in the United States, similar strains have been found in Britain, the Netherlands and parts of Africa. ${ }^{12-14}$ Given the capacity of the 25.2 megadalton plasmid for self transmission (in contrast to the betalactamase plasmids), such resistance is likely to disseminate rapidly. The plasmid had been thought to originate from the 24.5 megadalton conjugal gonococcal plasmid into which had been inserted the tet $M$ tetracycline resistance determinant. ${ }^{11}$ However, this view has been challenged recently. ${ }^{15}$

The speed with which PPNG and TRNG have disseminated illustrates a major difference between the impact of plasmid and chromosomal antibiotic resistance in the gonococcus. Even where tetracyclines are not used to treat gonorrhoea, their use in chlamydial infections and pelvic inflammatory dis- 
ease will probably assist the establishment of TRNG. TRNG can only be detected by antibiotic screening or plasmid analysis. Many laboratories will fail to recognise such strains.

Unfortunately there is no global surveillance system for following patterns of gonococcal resistance to antibiotics. Some countries carry out periodic or regular surveys of gonococcal resistance. In Britain laboratories are encouraged to send resistant gonococcal strains to the reference centres. While this system provides some idea of the prevalence of resistance, it does depend on the interest and goodwill of individual diagnostic laboratories and probably works best where screening methods are simplest as, for example, for PPNG. Other valuable sources of data are published surveys of resistance carried out by individual research groups. These will often highlight new problems or the spread of resistance to new geographical areas, but may not represent fully the national or regional picture.

Available data indicate that in North America and Europe, while the incidence of gonorrhoea is falling, the prevalence of antibiotic resistant strains is rising. In Britain resistant gonococci are still isolated relatively rarely in most STD clinics and treatment with oral or injectable penicillins remains appropriate. However, TRNG have been isolated and there are recent reports of treatment failures of ciprofloxacin associated with reduced in vitro susceptibility to this and other 4-quinolones. ${ }^{78}$

Gonococcal rates of resistance to antibiotics appear to be highest in Africa, South East Asia and parts of the Pacific. It is in these regions where the lack of any regular data collection about patterns of gonococcal resistance and the possible emergence of new forms of resistance is most serious. Without such information it is impossible to think about coherent strategies for controlling the spread of resistant strains and maintaining the efficacy of current antimicrobial therapies.

The problem is two-fold. First, lack of resources and expertise. Adequate facilities, training and support need to be provided to enable a few laboratory centres in these areas to carry out systematic gonococcal susceptibility testing. Second, the establishment of a world wide network into which all laboratories with such data can feed their information and receive in return a timely summary of trends world wide. The need for some such system of surveillance for gonococcal susceptibility to antimicrobial agents has been stated repeatedly by WHO working groups and expert committees and the WHO is the ideal organisation to provide such co-ordination. There are precedents with other micro-organisms.

At present third generation cephalosporins and related compounds, spectinomycin and fluoroquinolones provide good cover for resistant strains of
$N$ gonorrhoeae. However, there are actual or potential resistance problems with all three, which without careful control will grow. At present there is no new group of antimicrobial agents which is likely to extend our capacity to treat highly resistant gonococci. We have to make the best use of what is already available. Furthermore, the appearance of TRNG shows that we cannot necessarily predict future resistance problems. I find two possible future developments particularly disturbing. First, the appearance of plasmid-mediated, low level, enzymic resistance to spectinomycin. Second, the emergence of new beta lactamases in the gonococcus which inactivate antibiotics like ceftriaxone. At present this is idle speculation. I hope it remains so.

CHARLES SF EASMON

Department of Medical Microbiology St Mary's Hospital Medical School Imperial College of Science, Technology and Medicine

1 Current treatments in the control of sexually transmitted diseases. Report of a WHO Consultative Group 1982. WHO/ VDT/83.433.

2 STD treatment strategies. WHO Consultation on development of sexually transmitted disease Treatment Strategies 1989. WHO/VDT/89.477.

3 Sparling PF, Sarubbi FA, Blackman E. Inheritance of low-level resistance to penicillin, tetracycline, and chloroamphenicol in Neisseria gonorrhoeae, J Bacteriol 1975;124:740-9.

4 Maier TW, Zubrzycki L, Coyle MG. Genetic analysis in Neisseria gonorrhoeae: identification and linkage relationships of loci controlling drug resistance. Antimicrob Agents Chemother 1975;7:676-81.

5 Dougherty TJ, Koller AE, Tomasz A. Penicillin-binding proteins of penicillin-susceptible and intrinsically resistant Neisseria gonorrhoeae. Antimicrob Agents Chemother 1980;18:730-7.

6 Wagenvoort JHT, van der Willigen $A H$, van Vliet HJA, Michel MF, van Klingeren B. Resistance of Neisseria gonorrhoeae to enoxacin. J Antimicrob Chemother 1986;18:429.

7 Gransden WF, Warren CA, Phillips I, Hodges M, Barlow D. Decreased susceptibility of Neisseria gonorrhoeae to ciprofloxacin. Lancet 1990;1:51.

8 Jephcott AE, Turner A. Ciprofloxacin resistance in gonococci. Lancet 1990;1:165.

9 Phillips I. Penicillinase-producing penicillin resistant gonococcus. Lancet 1976;ii:656-7.

10 Ashford WA, Golash RG, Hemming VG. Penicillinase producing Neisseria gonorrhoeae. Lancet 1976;ii:657-8.

11 Centers for Disease Control. Tetracycline resistant Neisseria gonorrhoeae. Georgia, Pennsylvania, New Hampshire MMWR 1986.

12 Morse SA, Johnson SR, Biddle JW, Roberts MC. High level tetracycline resistance in Neisseria gonorrhoeae is the result of the acquisition of the streptococcal tet $M$ determinant. $J$ Infect Dis 1986;30:664-70.

13 Roberts MC, Wagenvoort JHT, van Klingeren B, Knapp JS. tet $M$ and $\beta$-lactamase containing Neisseria gonorrhoeae (tetracycline resistant and penicillinase-producing) in the Netherlands. Antimicrob Agents Chemother 1988;32:158.

14 Waugh MA, Lacey CJN, Hawkey PM, Heritage J, Turner A, Jephcott AE. Spread of Neisseria gonorrhoeae resistant to tetracycline outside the United States of America. Br Med J 1988;296:898.

15 Gascoyne DM, Heritage J, Hawkey PM. The $25 \cdot 2 \mathrm{MDa}$ tetracycline-resistance plasmid is not derived from the 24.5 MDa conjugative plasmid of Neisseria gonorrhoeae. J Antimicrob Chemother 1990;25:39-47. 